\title{
The dynamics of Baroclinic Zonal jets
}

\author{
Article
}

Published Version

Williams, P. D. and Kelsall, C. W. (2015) The dynamics of Baroclinic Zonal jets. Journal of the Atmospheric Sciences, 72 (3). pp. 1137-1151. ISSN 1520-0469 doi: https://doi.org/10.1175/JAS-D-14-0027.1 Available at https://centaur.reading.ac.uk/39399/

It is advisable to refer to the publisher's version if you intend to cite from the work. See Guidance on citing.

Published version at: http://dx.doi.org/10.1175/JAS-D-14-0027.1

To link to this article DOI: http://dx.doi.org/10.1175/JAS-D-14-0027.1

Publisher: American Meteorological Society

All outputs in CentAUR are protected by Intellectual Property Rights law, including copyright law. Copyright and IPR is retained by the creators or other copyright holders. Terms and conditions for use of this material are defined in the End User Agreement.

\section{www.reading.ac.uk/centaur}

\section{CentAUR}

Central Archive at the University of Reading

Reading's research outputs online 


\title{
The Dynamics of Baroclinic Zonal Jets*
}

\author{
PAUl D. Williams AND CHRistopher W. Kelsall \\ Department of Meteorology, University of Reading, Reading, United Kingdom
}

(Manuscript received 12 February 2014, in final form 18 November 2014)

\begin{abstract}
Multiple alternating zonal jets are a ubiquitous feature of planetary atmospheres and oceans. However, most studies to date have focused on the special case of barotropic jets. Here, the dynamics of freely evolving baroclinic jets are investigated using a two-layer quasigeostrophic annulus model with sloping topography. In a suite of 15 numerical simulations, the baroclinic Rossby radius and baroclinic Rhines scale are sampled by varying the stratification and root-mean-square eddy velocity, respectively. Small-scale eddies in the initial state evolve through geostrophic turbulence and accelerate zonally as they grow in horizontal scale, first isotropically and then anisotropically. This process leads ultimately to the formation of jets, which take about 2500 rotation periods to equilibrate. The kinetic energy spectrum of the equilibrated baroclinic zonal flow steepens from a -3 power law at small scales to a -5 power law near the jet scale. The conditions most favorable for producing multiple alternating baroclinic jets are large baroclinic Rossby radius (i.e., strong stratification) and small baroclinic Rhines scale (i.e., weak root-mean-square eddy velocity). The baroclinic jet width is diagnosed objectively and found to be 2.2-2.8 times larger than the baroclinic Rhines scale, with a best estimate of 2.5 times larger. This finding suggests that Rossby wave motions must be moving at speeds of approximately 6 times the turbulent eddy velocity in order to be capable of arresting the isotropic inverse energy cascade.
\end{abstract}

\section{Introduction}

Zonal jets in planetary atmospheres and oceans affect the transport of heat, momentum, and tracers. Satellite observations show clear evidence of multiple alternating zonal jets extending from the equator to the polar regions in the atmospheres of Jupiter (Limaye 1986) and Saturn (Sanchez-Lavega et al. 2000). In numerical simulations of the atmospheres of Jupiter, Saturn, Uranus, and Neptune, jets are found to emerge spontaneously from random initial conditions, in good agreement with observations in all four cases (Cho and Polvani 1996b). The existence of multiple alternating zonal jets in Earth's oceans has been inferred from sea surface heights measured by satellite altimetry, and similar jets are admitted in high-resolution ocean simulations (Maximenko et al.

\footnotetext{
* Supplemental information related to this paper is available at the Journals Online website: http://dx.doi.org/10.1175/JAS-D-140027.s1.

Corresponding author address: Paul D. Williams, Department of Meteorology, University of Reading, Earley Gate, Reading RG6 6BB, United Kingdom.

E-mail: p.d.williams@reading.ac.uk
}

2005). The zonal energy spectra in the terrestrial ocean and the atmospheres of the giant planets are found to be similar (Galperin et al. 2004), suggesting that the jets are governed by the same dynamics.

The generally accepted basic mechanism for the formation of zonal jets was proposed by Rhines (1975). In two-dimensional turbulent flows, there is an inverse (upscale) energy cascade, which is isotropic at small scales. The magnitude of the zonal phase speed of Rossby waves increases with wavelength, so the accumulation of energy at larger scales gradually causes Rossby wave motions to become more significant over time. Eventually, the Rossby waves are long enough that they move faster than the root-mean-square velocity associated with the turbulent eddy field. The Rossby waves then dominate the flow, arresting the isotropic inverse energy cascade and introducing a preference for the zonal elongation of eddies and ultimately the formation of jets. Several alternative interpretations of the formation of zonal jets have been proposed and developed, including descriptions based on nonequilibrium statistics (e.g., Bakas and Ioannou 2013). Jet formation may occur even in flows without cascading turbulent eddies through the self-maintenance of a potential vorticity staircase (e.g., Dunkerton and Scott 2008). 
The formation of zonal jets has been studied extensively in both numerical simulations (e.g., Maltrud and Vallis 1991; Huang and Robinson 1998; Sukoriansky et al. 2007; Dritschel and Scott 2011) and laboratory experiments (e.g., Sommeria et al. 1989; Read et al. 2007; Afanasyev et al. 2012; Di Nitto et al. 2013). The numerical and laboratory approaches both allow controlled and repeatable experiments to be performed in a wide range of dynamical regimes. On a rotating sphere, the potential vorticity gradient that is required for zonation is supplied by the latitudinal gradient of planetary vorticity. On a rotating platform in the laboratory, however, the analog of the planetary vorticity gradient is zero. Therefore, the potential vorticity gradient must instead be supplied by a fluid thickness gradient. One option for producing this gradient is to use radially sloping topography, to supply what has been referred to as the "topographic beta effect" (e.g., Read et al. 2007). Another option is to exploit the parabolic shape that is taken up by the free surface of the fluid in solid-body rotation, to supply what has been referred to as the "quadratic beta effect" (e.g., Bouchet and Sommeria 2002), "polar beta effect" (e.g., Afanasyev et al. 2012), or "gamma effect" (e.g., Di Nitto et al. 2013).

In laboratory experiments, Sommeria et al. (1989) identified a planetary eastward jet in a rotating annulus with an inner radius of $10.8 \mathrm{~cm}$ and an outer radius of $43.2 \mathrm{~cm}$. This experiment used a topographic beta effect, with fluid pumped radially inward from a ring of sources to a ring of sinks. Bastin and Read (1998) identified zonal jets in a rotating cylinder with a radius of $10.2 \mathrm{~cm}$ (see also Bastin and Read 1997). This experiment used a topographic beta effect, with small-scale turbulence excited by Ohmic heat dissipation within the electrolytic working fluid. Slavin and Afanasyev (2012) and Afanasyev et al. (2012) identified zonal jets in rotating cylinders with radii of 55 and $65 \mathrm{~cm}$. These experiments used a gamma effect, with small-scale turbulence excited locally by a radially oriented heating element that was immersed within the working fluid. Di Nitto et al. (2013) identified zonal jets in rotating square tanks with side lengths of 40 and $68 \mathrm{~cm}$. These experiments used a gamma effect, with small-scale turbulence excited by electromagnetic Lorentz forces associated with a horizontal array of permanent magnets. Most recently, Smith et al. (2014) identified multiple zonal jets in a differentially heated rotating annulus with an inner radius of $20.0 \mathrm{~cm}$ and an outer radius of $48.75 \mathrm{~cm}$. This experiment used both a topographic beta effect and a gamma effect, with geostrophic turbulence excited by baroclinic instability. Because of the relatively small geometric dimensions of all these experiments, they have been unable to access the high Reynolds numbers and low Ekman numbers that are relevant to zonation in planetary atmospheres and oceans.

In an attempt to access dynamical regimes at higher Reynolds numbers and lower Ekman numbers, Read et al. $(2004,2007)$ performed laboratory experiments on the Coriolis platform in Grenoble, France. This rotating turntable has a diameter of $14 \mathrm{~m}$. The experiments were performed in a rotating annulus with an inner radius of $2 \mathrm{~m}$ and an outer radius of $6.5 \mathrm{~m}$. The bottom of the annular tank sloped downward with increasing radius, providing a topographic beta effect. Convective forcing was applied by spraying saline water onto the free surface of the relatively fresh water in the tank, to generate small-scale convective cells and associated turbulent eddies. The Reynolds number (based on the jet scale) was estimated to exceed $2 \times 10^{3}$, which is at least an order of magnitude greater than the Reynolds numbers that had been achieved in previous laboratory experiments, and the Ekman number was estimated to be less than $2 \times 10^{-5}$. There was clear evidence of zonal jet formation on the Rhines scale, although the jets meandered substantially and occasionally split and merged, unlike the relatively steady jets on Jupiter and Saturn.

Most numerical and laboratory studies to date have focused on the special case of jets that are barotropic [see Lee (2005) and Smith et al. (2014) for exceptions]. However, the planetary atmospheres and oceans that exhibit multiple alternating zonal jets are stratified fluids, with a spectrum of excited baroclinic modes in addition to the barotropic mode. Zonal jets in the baroclinic modes differ dynamically from zonal jets in the barotropic mode, because, in contrast to barotropic Rossby waves, the magnitude of the zonal phase speed of baroclinic Rossby waves does not continue to increase without limit as their wavelength increases. This property of baroclinic Rossby waves yields a condition for baroclinic zonal jets to form (Scott 2010), whereas barotropic zonal jets form unconditionally.

The goal of the present paper is to investigate the dynamics of freely evolving baroclinic zonal jets. In particular, we wish to study the dependence of the structure of the jets on two key length scales: the baroclinic Rossby radius and the baroclinic Rhines scale. To this end, we use a two-layer quasigeostrophic annulus model with sloping topography to perform numerical simulations in different dynamical regimes. The simulations are initialized with a field of random small-scale isotropic eddies, from which jets can form if conditions allow. The layout of the paper is as follows. First, the physical and numerical aspects of the model are stated and the suite of simulations is described (section 2). Then, the approach to equilibrium is analyzed in a case-study simulation before the equilibrium states are analyzed in the entire suite of simulations 
(section 3). The paper concludes with a summary and discussion (section 4).

\section{Methodology}

In this section, we give details of the physics and numerics of the model before describing the suite of simulations that have been performed.

\section{a. Physical aspects}

Figure 1 shows a schematic diagram of the system being modeled. The rotating annulus has inner radius $a$, outer radius $b$, and average height $2 H$. It contains equal volumes of two incompressible fluids with densities $\rho_{i}$, where $i=1$ refers to the upper layer and $i=2$ refers to the lower layer. The average density is $\bar{\rho}=\left(\rho_{1}+\rho_{2}\right) / 2$ and the density difference is $\Delta \rho=\rho_{2}-\rho_{1}$. The upper and lower boundaries are axisymmetric and slope radially with gradients of $+s$ and $-s$, respectively. The system rotates about the axis of symmetry with an angular velocity of magnitude $\Omega$ in the anticlockwise direction, as viewed from above. The acceleration due to gravity has magnitude $g$. We choose annular geometry for the simulations in order to facilitate comparisons with previous laboratory experiments. We note that channel simulations are not well suited to this purpose, because the periodic channel has additional shift-reflect symmetries compared to the annulus (Cattaneo and Hart 1990), causing some of the wave-wave interactions that are present in the annulus to be absent (Kwon and Mak 1988).

We model the system using the quasigeostrophic equations, which are relatively inexpensive to integrate numerically. Ageostrophic motions, including inertiagravity waves, are absent from quasigeostrophic models but are unimportant for jet formation. Quasigeostrophic models have been used previously to simulate jet formation (e.g., Panetta 1993; Marcus and Lee 1998; Thompson and Young 2007) and have been found to remain reasonably accurate even outside the strict quasigeostrophic regime (Williams et al. 2010).

We define $f=2 \Omega$ to be the Coriolis parameter and $g^{\prime}=g \Delta \rho / \bar{\rho}$ to be the reduced gravity. In layer $i$, we write the streamfunction as $\psi_{i}$ and the potential vorticity under the quasigeostrophic approximation as $\left(f+q_{i}\right) / H$. Then, in cylindrical polar coordinates defined by $(r, \theta)$, the quasigeostrophic equations for this system are

$$
\begin{aligned}
& \frac{\partial q_{1}}{\partial t}-\frac{1}{r} \frac{\partial \psi_{1}}{\partial \theta} \frac{\partial q_{1}}{\partial r}+\frac{1}{r} \frac{\partial \psi_{1}}{\partial r} \frac{\partial q_{1}}{\partial \theta}=0 \quad \text { and } \\
& \frac{\partial q_{2}}{\partial t}-\frac{1}{r} \frac{\partial \psi_{2}}{\partial \theta} \frac{\partial q_{2}}{\partial r}+\frac{1}{r} \frac{\partial \psi_{2}}{\partial r} \frac{\partial q_{2}}{\partial \theta}=0,
\end{aligned}
$$

where

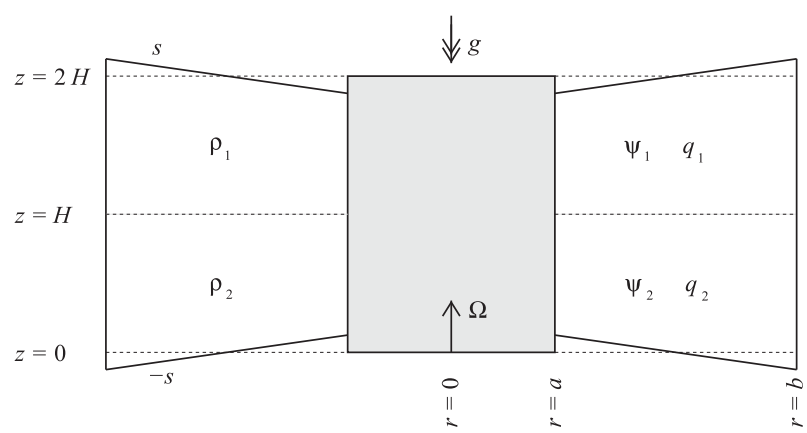

FIG. 1. Schematic diagram of the rotating annulus being modeled (not to scale). See section 2a for details.

$$
\begin{aligned}
& q_{1}=\nabla^{2} \psi_{1}+\frac{f^{2}}{g^{\prime} H}\left(\psi_{2}-\psi_{1}\right)-\frac{f s r}{H} \quad \text { and } \\
& q_{2}=\nabla^{2} \psi_{2}-\frac{f^{2}}{g^{\prime} H}\left(\psi_{2}-\psi_{1}\right)-\frac{f_{s r}}{H} .
\end{aligned}
$$

Equations (1) and (2) express the conservation of potential vorticity following fluid parcels. Equations (3) and (4) indicate that the potential vorticity has contributions from the local relative vorticity (the $\nabla^{2}$ terms) and from vortex stretching associated both with internal interface height perturbations (the $\psi_{2}-\psi_{1}$ terms) and with the sloping base and lid (the $r$ terms). The potential vorticity field in each layer is initialized with small-scale noise of amplitude $q_{0}$ (see section $2 \mathrm{~b}$ ).

In the limit of negligible advection, (1)-(4) become linear and permit barotropic and baroclinic Rossby waves with zonal phase speeds of

$$
\begin{aligned}
& c_{\mathrm{bt}}=\frac{-\beta}{k^{2}+l^{2}} \quad \text { and } \\
& c_{\mathrm{bc}}=\frac{-\beta}{k^{2}+l^{2}+1 / L_{\mathrm{Ro}, \mathrm{bc}}^{2}},
\end{aligned}
$$

respectively. Here, $\beta=f_{s} / H$ is the topographic beta effect, $k$ and $l$ are the zonal (i.e., azimuthal) and meridional (i.e., radial) wave vectors, respectively, and

$$
L_{\mathrm{Ro}, \mathrm{bc}}=\sqrt{\frac{g^{\prime} H}{2 f^{2}}}
$$

is the baroclinic Rossby radius. Note that as $k \rightarrow 0$ and $l \rightarrow 0,\left|c_{\mathrm{bt}}\right|$ increases without limit, but $\left|c_{\mathrm{bc}}\right|$ saturates at $\beta L_{\mathrm{Ro}, \mathrm{bc}}^{2}$. Formulas for the barotropic and baroclinic Rhines scales, which are defined to be the distance between adjacent positive and negative jet maxima in the barotropic and baroclinic flow, are traditionally derived by equating the magnitudes of the zonal phase speeds in 
(5) and (6) to the root-mean-square eddy velocity $u_{\text {rms }}$ and setting $k=l$. Note that the desired length scale is half of the meridional wavelength (i.e., half of $2 \pi / l$ ), because each meridional wavelength contains one positive (prograde) jet and one negative (retrograde) jet. This exercise yields

$$
\begin{aligned}
& L_{\mathrm{Rh}, \mathrm{bt}}=\pi \sqrt{\frac{2 u_{\mathrm{rms}}}{\beta}} \text { and } \\
& L_{\mathrm{Rh}, \mathrm{bc}}=\pi \sqrt{\frac{2 u_{\mathrm{rms}}}{\beta-u_{\mathrm{rms}} / L_{\mathrm{Ro}, \mathrm{bc}}^{2}}} .
\end{aligned}
$$

The dependence of $L_{\mathrm{Rh}, \mathrm{bc}}$ on $u_{\mathrm{rms}}$ in (9) is qualitatively different from the dependence of $L_{\mathrm{Rh}, \mathrm{bt}}$ on $u_{\mathrm{rms}}$ in (8). In particular, if $u_{\mathrm{rms}}>\beta L_{\mathrm{Ro}, \mathrm{bc}}^{2}$, then $L_{\mathrm{Rh}, \mathrm{bc}}$ is undefined. In this case, eddies can grow arbitrarily large through the isotropic inverse energy cascade without baroclinic Rossby wave motions ever becoming important. This is therefore a turbulence-dominated regime, in which the baroclinic flow remains isotropic. If $u_{\mathrm{rms}} \leqq \beta L_{\mathrm{Ro}, \mathrm{bc}}^{2}$, then $L_{\mathrm{Rh}, \mathrm{bc}}$ is defined and satisfies $L_{\mathrm{Rh}, \mathrm{bc}} \gg L_{\mathrm{Ro}, \mathrm{bc}}$. In this case, once eddies have grown isotropically to the scale of $L_{\mathrm{Rh}, \mathrm{bc}}$, baroclinic Rossby wave motions are just starting to become significant, but further eddy growth does not lead to a zonal acceleration, because the phase speed limit has already been reached. This is therefore a regime in which the baroclinic flow remains approximately isotropic. If $u_{\mathrm{rms}} \ll \beta L_{\mathrm{Ro}, \mathrm{bc}}^{2}$, then $L_{\mathrm{Rh}, \mathrm{bc}}$ is defined and satisfies $L_{\mathrm{Rh}, \mathrm{bc}} \ll L_{\mathrm{Ro} \text {,bc. }}$. In this case, once eddies have grown isotropically to the scale of $L_{\mathrm{Rh}, \mathrm{bc}}$, further zonal growth leads to a zonal acceleration, which leads to further zonal growth, etc. This is therefore a jet-dominated regime, in which the baroclinic flow is strongly anisotropic. We anticipate an intermediate regime defined by $u_{\mathrm{rms}}<\beta L_{\mathrm{Ro}, \mathrm{bc}}^{2}$ but $u_{\mathrm{rms}} \approx \beta L_{\mathrm{Ro}, \mathrm{bc}}^{2}$ and $u_{\mathrm{rms}} \nless \beta L_{\mathrm{Ro} \text {, bc }}^{2}$, in which the baroclinic flow develops jets that are transient and undulatory because of interactions with the relatively strong eddies.

The nondimensional parameter $\mathrm{Rh}=u_{\mathrm{rms}} /\left(\beta L_{\mathrm{Ro}, \mathrm{bc}}^{2}\right)$ has been referred to as the Rhines number (e.g., Saito and Ishioka 2013). Using this terminology to summarize the above dynamical regimes, we expect the equilibrated baroclinic flow to be isotropic if $\mathrm{Rh}>1$; to be approximately isotropic if $\mathrm{Rh} \cong 1$; to contain zonal jets that are transient and undulatory if $\mathrm{Rh}<1$ but $\mathrm{Rh} \approx 1$ and $\mathrm{Rh} \nless 1$; and to contain zonal jets that are robust if $\mathrm{Rh} \ll 1$.

Table 1 lists the physical parameter values that are used in the present simulations. The annulus dimensions, total fluid depth, average fluid density, topographic beta effect, and rotation rate are all fixed at values chosen to match the main laboratory experiments of Read et al. (2004, 2007), because these are the laboratory experiments that
TABLE 1. Summary of the physical parameters used in the simulations. See section 2a for details.

\begin{tabular}{lcl}
\hline \multicolumn{1}{c}{ Description } & Parameter & \multicolumn{1}{c}{ Value } \\
\hline Inner radius of annulus & $a$ & $2.0 \mathrm{~m}$ \\
Outer radius of annulus & $b$ & $6.5 \mathrm{~m}$ \\
Thickness of each fluid layer & $H$ & $0.275 \mathrm{~m}$ \\
Average density of fluid & $\bar{\rho}$ & $1000.0 \mathrm{~kg} \mathrm{~m}^{-3}$ \\
Radial slope of lid & $+s$ & 0.04 \\
Radial slope of base & $-s$ & -0.04 \\
Rotation rate & $\Omega$ & $2 \pi / 40 \mathrm{rad} \mathrm{s}^{-1}$ \\
Acceleration due to gravity & $g$ & $9.81 \mathrm{~m} \mathrm{~s}^{-2}$ \\
Density difference & $\Delta \rho$ & $($ see Table 3) \\
Amplitude of initial condition & $q_{0}$ & $($ see Table 3) \\
\hline
\end{tabular}

have come the closest to accessing the high Reynolds numbers and low Ekman numbers that are relevant to zonation in planetary atmospheres and oceans. The density difference and initial-condition amplitude are used as control parameters (see section 2c). Note that the equilibrium height of the internal interface between the two fluid layers will take up a parabolic $\Omega^{2} r^{2} /(2 g)$ shape that is associated with centrifugal effects in solid-body rotation. With the parameter values given in Table 1 , however, the height increment of the parabolic shape across the annular gap is only $\Omega^{2}\left(b^{2}-a^{2}\right) /(2 g) \approx 5 \mathrm{~cm}$, whereas the total fluid depth changes by $2 s(b-a) \approx 36 \mathrm{~cm}$. Therefore, the contribution made by the parabolic shape to the potential vorticity through vortex stretching has been justifiably neglected in (3) and (4).

\section{b. Numerical aspects}

To integrate (1)-(4) numerically, we use version 1.3 of the Quasi-Geostrophic Model for Investigating Rotating Fluids Experiments (QUAGMIRE), which was developed by Williams et al. (2009) and has been used in various studies of the rotating annulus (e.g., Williams et al. 2003, 2004, 2005). The model integrates the equations with respect to time using a hybrid numerical scheme that is partly finite difference and partly spectral. Given $q_{i}$ and $\psi_{i}$ at any time, (1) and (2) are invoked to step $q_{i}$ forward in time in physical space, using finite differences and a leapfrog step. Then, (3) and (4) are invoked to invert $q_{i}$ at the future time and obtain the corresponding $\psi_{i}$. The inversion is done after transforming both to Fourier modes in the zonal direction and to the barotropic and baroclinic modes in the vertical direction. The inversion imposes boundary conditions at $r=a$ and $r=b$ of

$$
\begin{aligned}
\frac{\partial \psi_{i}}{\partial \theta} & =0 \quad \text { and } \\
\int_{0}^{2 \pi} \frac{\partial \psi_{i}}{\partial r} d \theta & =0
\end{aligned}
$$


TABLE 2. Summary of the numerical parameters used in the simulations. See section $2 b$ for details.

\begin{tabular}{llc}
\hline \multicolumn{1}{c}{ Description } & Parameter & Value \\
\hline Number of radial grid points & $N_{\text {rad }}$ & 128 \\
Number of azimuthal grid points & $N_{\text {azim }}$ & 1024 \\
Time step & $\Delta t$ & $1.0 \mathrm{~s}$ \\
Robert filter parameter & $\nu_{\mathrm{RA}}$ & 0.01 \\
Hyperdiffusion coefficient & $\nu_{\mathrm{PV}}$ & $1.0 \times 10^{-8} \mathrm{~m}^{2} \mathrm{~s}^{-1}$ \\
\hline
\end{tabular}

where (10) ensures that the sidewalls are impermeable to the flow, and (11) ensures that the zonal-mean zonal flow satisfies the no-slip condition at the sidewalls.

The numerical grid consists of $N_{\text {rad }}$ points in the radial direction (including one point on each of the boundaries at $r=a$ and $r=b$ ) and $N_{\text {azim }}$ points in the azimuthal direction. The time step is $\Delta t$. A Robert-Asselin filter (Asselin 1972) with parameter $\nu_{\mathrm{RA}}$ is applied to $q_{i}$ after each time step to suppress the $2 \Delta t$ computational mode of the leapfrog scheme, as is commonly done in numerical models (Williams 2009, 2011, 2013). For numerical stability, time-lagged hyperdiffusion is added to (1) and (2) with a coefficient of $\nu_{\mathrm{PV}}$. The hyperdiffusion takes the form of a second-order (Laplacian) operator acting on $q_{i}$. Because $q_{i}$ is related to the Laplacian of $\psi_{i}$ through (3) and (4), the hyperdiffusion is effectively a fourth-order (biharmonic) operator acting on $\psi_{i}$.

Table 2 lists the numerical parameter values that are used in the present simulations. The radial and azimuthal grid resolutions are chosen to make the grid boxes approximately square throughout the annular domain. Specifically, the radial grid spacing is $3.5 \mathrm{~cm}$ at all radii, and the azimuthal grid spacing varies from $1.2 \mathrm{~cm}$ at the inner boundary to $4.0 \mathrm{~cm}$ at the outer boundary. The time step is chosen to make the Courant number equal 0.1 , based on a root-mean-square velocity of $1 \mathrm{~mm} \mathrm{~s}^{-1}$ and a grid spacing of $1 \mathrm{~cm}$. The RobertAsselin filter parameter is chosen to damp the computational mode with an $e$-folding time of around $100 \mathrm{~s}$. The hyperdiffusion coefficient is chosen to damp gridscale features with an $e$-folding time of around $1000 \mathrm{~s}$.

Noting that the equilibrium solution to (1)-(4) subject to (10) and (11) is $\psi_{i}=0$ and $q_{i}=-f_{s r} / H$, the fields $q_{i}+$ $f s r / H$ are initialized by sampling randomly from a uniform distribution between $-q_{0}$ and $+q_{0}$ at each grid point. An isotropic two-dimensional bandpass filter is then applied in radius and azimuth to remove grid-scale and large-scale features. The filter shape is Gaussian in wavenumber space and permits wavelengths mainly between 5 and 10 times the grid spacing. Specifically, the Fourier coefficients of wavelengths outside this range are reduced by a factor of at least $e$. The result is an initial condition in which only a narrow spectral band of small-scale isotropic eddies is energized, as shown in

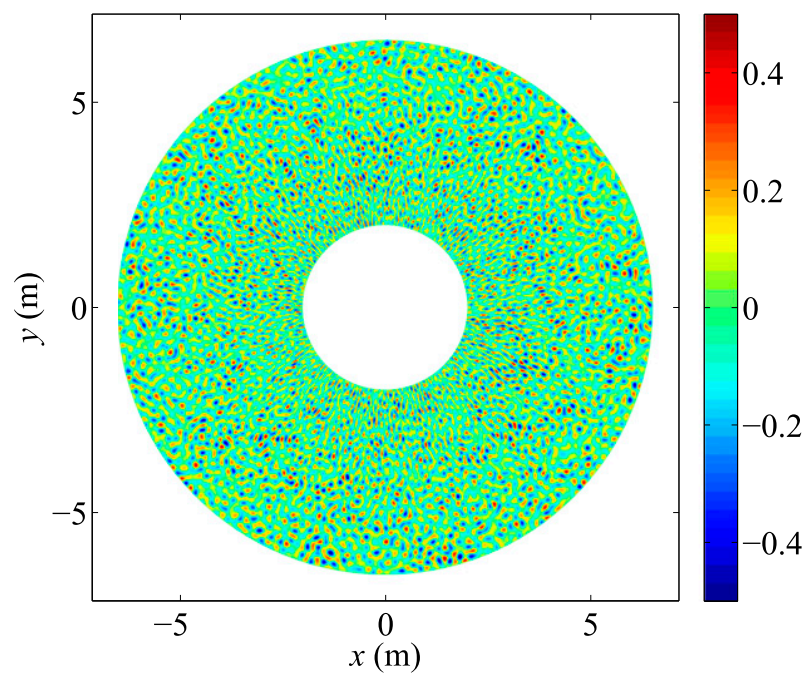

FIG. 2. An example of the initial condition on $q_{i}+f_{s} / H$ in either of the layers $\left(\mathrm{s}^{-1}\right)$. A noise field of amplitude $q_{0}=1.0 \mathrm{~s}^{-1}$ has been bandpass filtered to permit wavelengths mainly between 5 and 10 times the grid spacing, as described in section $2 \mathrm{~b}$.

Fig. 2. An independent noise realization is used in each layer to excite both the barotropic and baroclinic modes.

\section{c. Suite of simulations}

A suite of 15 numerical simulations has been performed to integrate the full nonlinear quasigeostrophic model in (1)-(4) subject to (10) and (11). The details of the simulations are listed in Table 3 . The first control parameter being varied is the density difference $\Delta \rho$, which directly controls the reduced gravity $g^{\prime}$ and indirectly controls the baroclinic Rossby radius $L_{\text {Ro,bc }}$ via (7). We choose three values for $\Delta \rho: 1.0,10.0$, and $100.0 \mathrm{~kg} \mathrm{~m}^{-3}$. We fix $\bar{\rho}=1000.0 \mathrm{~kg} \mathrm{~m}^{-3}$ for all the simulations, using $\rho_{1}=$ $999.5 \mathrm{~kg} \mathrm{~m}^{-3}$ and $\rho_{2}=1000.5 \mathrm{~kg} \mathrm{~m}^{-3}$ to obtain $\Delta \rho=$ $1.0 \mathrm{~kg} \mathrm{~m}^{-3} ; \rho_{1}=995.0 \mathrm{~kg} \mathrm{~m}^{-3}$ and $\rho_{2}=1005.0 \mathrm{~kg} \mathrm{~m}^{-3}$ to obtain $\Delta \rho=10.0 \mathrm{~kg} \mathrm{~m}^{-3}$; and $\rho_{1}=950.0 \mathrm{~kg} \mathrm{~m}^{-3}$ and $\rho_{2}=$ $1050.0 \mathrm{~kg} \mathrm{~m}^{-3}$ to obtain $\Delta \rho=100.0 \mathrm{~kg} \mathrm{~m}^{-3}$.

The second control parameter being varied is the amplitude $q_{0}$ of the initial condition, which directly controls the root-mean-square velocity $u_{\mathrm{rms}}$ of the turbulent eddy field and indirectly controls the baroclinic Rhines scale $L_{\mathrm{Rh}, \mathrm{bc}}$ via (9). We choose five values for $q_{0}$ : 0.04 , $0.09,0.16,0.25$, and $0.36 \mathrm{~s}^{-1}$. Because $u_{\mathrm{rms}}$ is proportional to $q_{0}$, these values imply that $L_{\mathrm{Rh}, \mathrm{bc}}$ is being sampled uniformly if $u_{\mathrm{rms}} \ll \beta L_{\mathrm{Ro}, \mathrm{bc}}^{2}$. The corresponding rootmean-square eddy velocities are found to be approximately $0.15,0.38,0.72,1.15$, and $1.66 \mathrm{~mm} \mathrm{~s}^{-1}$, respectively (see Table 3).

We have run one simulation for each combination of $\Delta \rho$ and $q_{0}$. Each simulation was run for 100000 s (27.8 h) of simulated time and required $3.7 \mathrm{~h}$ of elapsed time on a desktop personal computer with a $3.1-\mathrm{GHz}$ processor. 
TABLE 3. Summary of the 15 numerical simulations performed. The two control parameters are the density difference $\Delta \rho$ and the initialcondition amplitude $q_{0}$. The root-mean-square velocity $u_{\mathrm{rms}}$ and the equilibrated baroclinic jet width are diagnosed as described in section 3a. The baroclinic Rossby radius $L_{\mathrm{Ro}, \mathrm{bc}}$ is calculated using (7), and the baroclinic Rhines scale $L_{\mathrm{Rh}, \mathrm{bc}}$ is calculated using (9). The baroclinic Rhines scale is undefined if $u_{\mathrm{rms}}>\beta L_{\mathrm{Ro}, \mathrm{bc}}^{2}$, in which case the baroclinic jet width is not diagnosed.

\begin{tabular}{ccccccc}
\hline \hline Simulation number & $\Delta \rho\left(\mathrm{kg} \mathrm{m}^{-3}\right)$ & $q_{0}\left(\mathrm{~s}^{-1}\right)$ & Diagnosed $u_{\mathrm{rms}}\left(\mathrm{mm} \mathrm{s}^{-1}\right)$ & $L_{\mathrm{Ro}, \mathrm{bc}}(\mathrm{m})$ & $L_{\mathrm{Rh}, \mathrm{bc}}(\mathrm{m})$ & Diagnosed baroclinic jet width $(\mathrm{m})$ \\
\hline 1 & 1.0 & 0.04 & 0.14 & 0.12 & 0.28 & $1.08 \pm 0.63$ \\
2 & 1.0 & 0.09 & 0.38 & 0.12 & 0.64 & $0.85 \pm 0.41$ \\
3 & 1.0 & 0.16 & 0.71 & 0.12 & - & - \\
4 & 1.0 & 0.25 & 1.13 & 0.12 & - & - \\
5 & 1.0 & 0.36 & 1.65 & 0.12 & - & $0.58 \pm 0.12$ \\
6 & 10.0 & 0.04 & 0.16 & 0.37 & 0.26 & $1.22 \pm 0.38$ \\
7 & 10.0 & 0.09 & 0.38 & 0.37 & 0.42 & $2.56 \pm 0.42$ \\
8 & 10.0 & 0.16 & 0.71 & 0.37 & 0.59 & 0.57 \\
9 & 10.0 & 0.25 & 1.16 & 0.37 & 0.78 & $0.35 \pm 0.79$ \\
10 & 10.0 & 0.36 & 1.68 & 0.37 & 1.00 & $0.59 \pm 0.08$ \\
11 & 100.0 & 0.04 & 0.16 & 1.17 & 0.26 & $0.87 \pm 0.08$ \\
12 & 100.0 & 0.09 & 0.39 & 1.17 & 0.41 & $1.41 \pm 0.27$ \\
13 & 100.0 & 0.16 & 0.74 & 1.17 & 0.57 & $1.67 \pm 0.52$ \\
15 & 100.0 & 0.25 & 1.66 & 1.17 & 0.72 & $2.08 \pm 0.41$
\end{tabular}

For simulations $1-5$, the baroclinic Rossby radius is sufficiently short that $u_{\text {rms }} \approx \beta L_{\text {Ro,bc }}^{2}$; therefore, we expect the equilibrated baroclinic flow to be approximately isotropic. For simulations $6-10$, the baroclinic Rossby radius takes an intermediate value such that $u_{\mathrm{rms}}<\beta L_{\mathrm{Ro}, \mathrm{bc}}^{2}$ but $u_{\mathrm{rms}} \approx \beta L_{\mathrm{Ro}, \mathrm{bc}}^{2}$ and $u_{\mathrm{rms}} \nless \beta L_{\mathrm{Ro}, \mathrm{bc}}^{2}$ (except perhaps in simulations 6 and 7); therefore, we expect the equilibrated baroclinic flow to exhibit transient and undulatory zonation. For simulations 11-15, the baroclinic Rossby radius is sufficiently long that $u_{\mathrm{rms}} \ll \beta L_{\mathrm{Ro}, \mathrm{bc}}^{2}$; therefore, we expect the equilibrated baroclinic flow to exhibit robust zonation.

There is a hierarchy of horizontal length scales in the simulations, as follows. The grid spacing is substantially smaller than the range of scales energized in the initial state, so the initial eddies are not efficiently dissipated by the numerical scheme or the hyperdiffusion. In turn, the scales energized in the initial state are typically smaller than the baroclinic Rhines scales, to allow an isotropic inverse cascade of energy to occur in the baroclinic flow before anisotropic effects become important. In turn, the baroclinic Rhines scales are substantially smaller than the width of the annular gap, to permit multiple alternating baroclinic jets to develop if conditions allow.

\section{Results}

In this section, we analyze the approach to equilibrium in a case-study simulation before analyzing the final equilibrium states in all 15 simulations.

\section{a. The approach to equilibrium}

We analyze the approach to equilibrium using simulation 11 from Table 3 as a case study. Snapshots of the baroclinic streamfunction $\left(\psi_{2}-\psi_{1}\right)$ at various times are shown in Fig. 3. The small-scale eddies that are present in the initial condition evolve through geostrophic turbulence and grow isotropically in horizontal scale for at least $5000 \mathrm{~s}$. The first signs of anisotropy are evident from about $10000 \mathrm{~s}$, when the eddies have started to arrange themselves with some zonal coherence. Eddy growth in the meridional direction appears to be largely arrested after about $25000 \mathrm{~s}$, but elongation in the zonal direction continues as eddies collide and merge. By $100000 \mathrm{~s}$, approximately eight alternating baroclinic zonal jets have become well established. The jets appear to have a spiral structure, in the sense that the radius of the jet maximum varies with azimuthal angle. Spiral structures have been noted previously in numerical simulations of jets (Cho and Polvani 1996a) but their origin is unclear. A movie showing the development of the jets in more detail (every 100s) is included in the supplemental material.

A Hovmöller (1949) azimuth-time diagram of the baroclinic streamfunction at midradius is shown in Fig. 4. The gradual upscale cascade of energy in the zonal direction is clear. The motion of individual eddies as they grow is always clockwise or retrograde (i.e., in the opposite direction to the rotation of the tank), as expected from the sign of the topographic beta effect. The flow structures experience a zonal acceleration as they grow in scale, consistent with the wavelength dependence of the phase speed of baroclinic Rossby waves in (6). By $100000 \mathrm{~s}$, the midradius baroclinic streamfunction is dominated by a large and rapidly moving structure with a zonal wavenumber of 1 .

A Hovmöller radius-time diagram of the baroclinic zonal-mean zonal velocity is shown in Fig. 5. Note that 
(a) $t=1000 \mathrm{~s}$

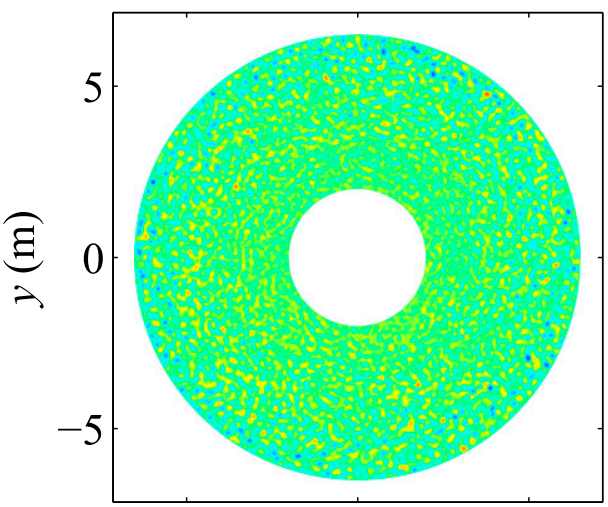

(c) $t=10000 \mathrm{~s}$

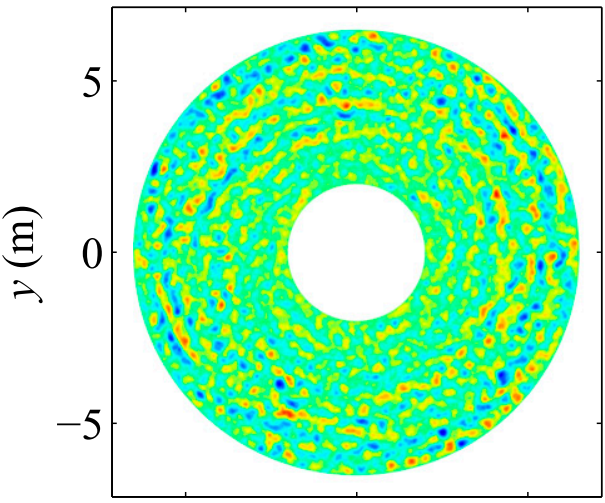

(e) $t=50000 \mathrm{~s}$

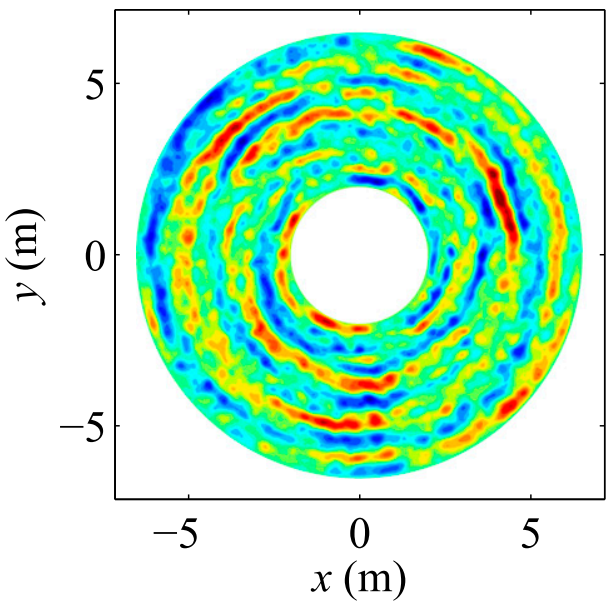

(b) $t=5000 \mathrm{~s}$

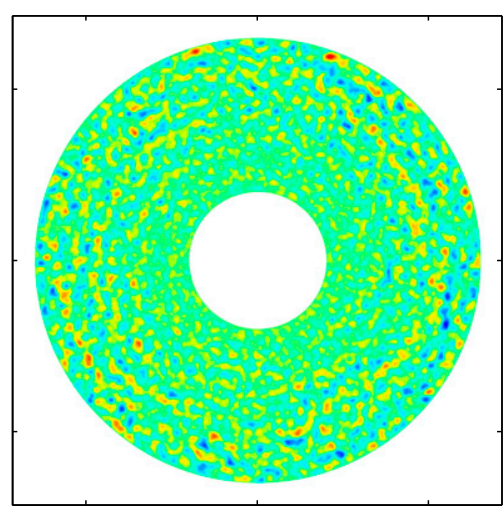

(d) $t=25000 \mathrm{~s}$

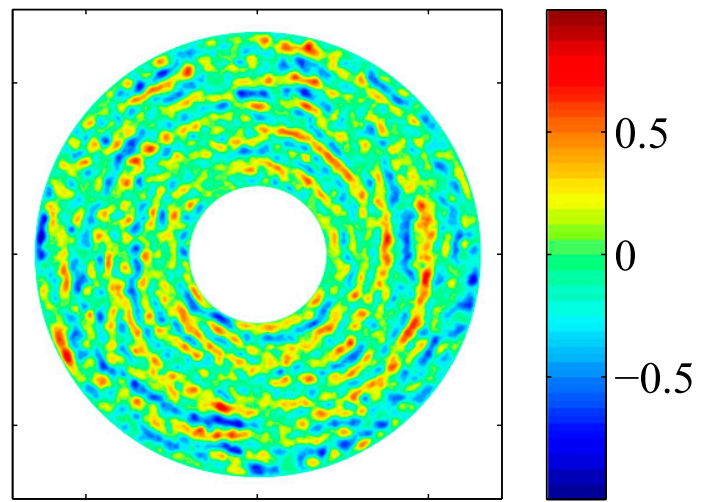

(f) $t=100000 \mathrm{~s}$

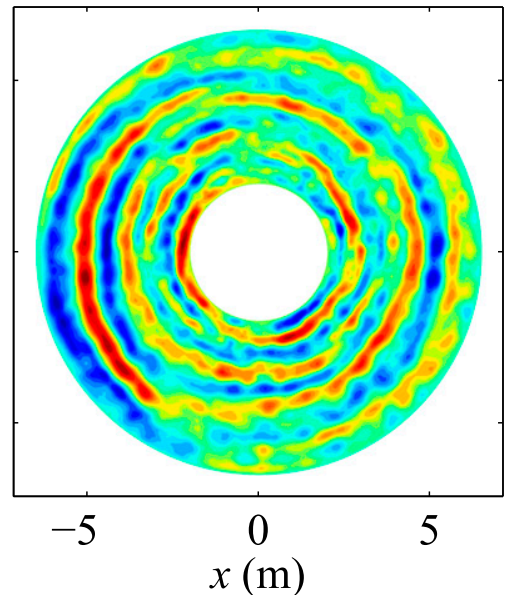

FIG. 3. Snapshots from simulation 11, showing the evolution of the baroclinic streamfunction $\left(\mathrm{m}^{2} \mathrm{~s}^{-1}\right)$ at the specified times.

the zonal-mean zonal velocity must be interpreted with some caution, because the spiral nature of the jets (see Fig. 3) implies a degree of cancellation between prograde and retrograde motions. Therefore, the zonal-mean jets are expected to be substantially weaker and narrower than the jets at any given azimuthal angle. Mindful of this caveat, around 13 alternating zonal-mean jets of strength up to about $0.1 \mathrm{~mm} \mathrm{~s}^{-1}$ emerge from the initial condition by $100000 \mathrm{~s}$. 


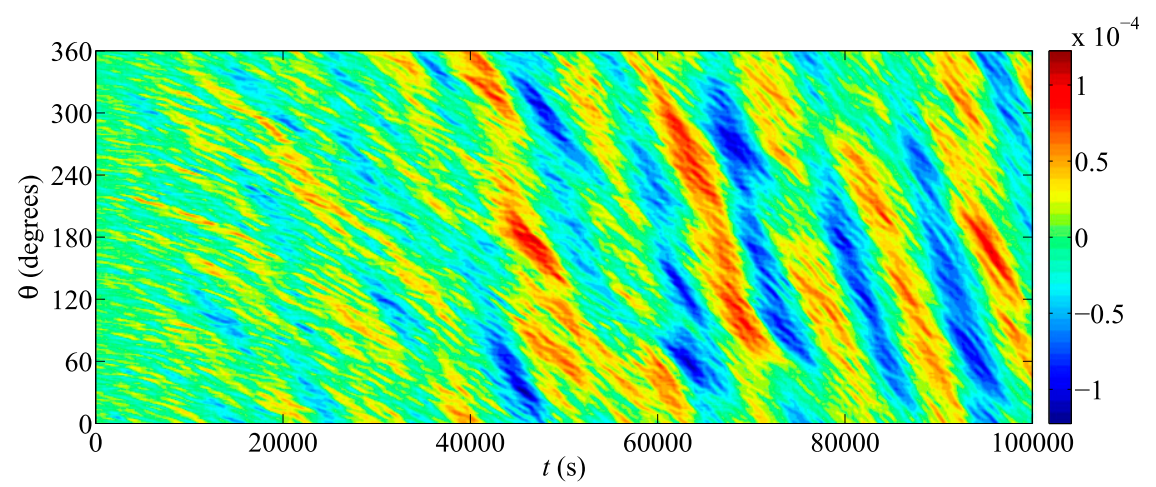

FIG. 4. A Hovmöller diagram from simulation 11, showing the evolution of the baroclinic streamfunction $\left(\mathrm{m}^{2} \mathrm{~s}^{-1}\right)$ at radius $r=(a+b) / 2$. The azimuthal angle $\theta=0^{\circ}$ refers to the " 3 o'clock" position, and $\theta$ increases in the anticlockwise direction.

Time series of the root-mean-square velocities are shown in Fig. 6. To expose the developing anisotropy, the total root-mean-square velocities are decomposed into their zonal and meridional components. Initially, the total root-mean-square velocity in each layer has equal contributions from the zonal and meridional components, indicating that the velocity field is isotropic. As the flow evolves, however, the meridional components decrease and the zonal components increase. The total root-mean-square velocities in each layer are approximately conserved. By averaging the total root-mean-square velocities over all times and both layers, the characteristic root-mean-square velocity for this simulation is diagnosed to be $0.16 \mathrm{~mm} \mathrm{~s}^{-1}$. An anisotropy index may be defined by subtracting the meansquared meridional velocity from the mean-squared zonal velocity and then dividing the result by their sum (e.g., Di Nitto et al. 2013). The anisotropy index is -1 if the flow is purely meridional, 0 if the flow is perfectly isotropic, and +1 if the flow is purely zonal. Time series of the anisotropy index in each layer are included in Fig. 6 . The anisotropy indices increase from 0 to 0.85 over the integration period, quantifying the emergence of the strongly zonal flow from the isotropic initial state.

The baroclinic zonal kinetic energy spectrum is shown in Fig. 7. The behavior of the smallest resolved scales is consistent with a -3 power law, as expected for twodimensional quasigeostrophic turbulence. However, the behavior for larger scales is more consistent with a -5 power law. The transition to a steep -5 power law has been noted in other studies (e.g., Galperin et al. 2001; Sukoriansky et al. 2002; Galperin et al. 2004). The -3 and -5 slopes are exhibited over relatively small ranges of meridional wavenumber, and the degree to which they are robust will not be pursued here. The peak of the spectrum occurs at the fourth-gravest meridional mode, which corresponds to eight alternating jets across the annular gap, consistent with Fig. 3f. There is some evidence of a nonphysical buildup of energy near the grid scale, which could be controlled by increasing the strength of the hyperdiffusion, but which is far too weak to jeopardize the stability of the numerical scheme.

An objective method for diagnosing the baroclinic jet width is presented in Fig. 8, using the flow in Fig. $3 \mathrm{f}$ for

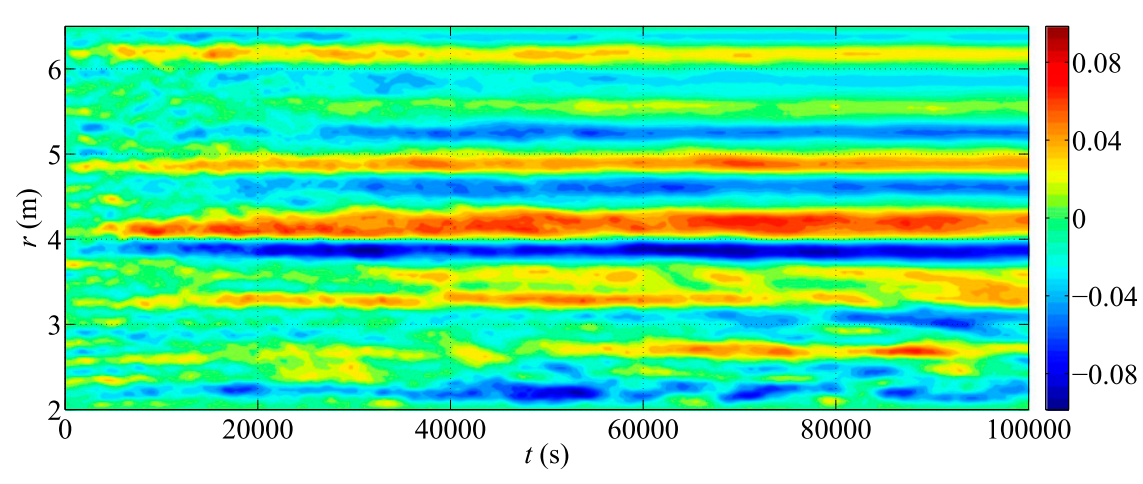

FIG. 5. A Hovmöller diagram from simulation 11, showing the evolution of the baroclinic zonal-mean zonal velocity $\left(\mathrm{mm} \mathrm{s}^{-1}\right)$. 


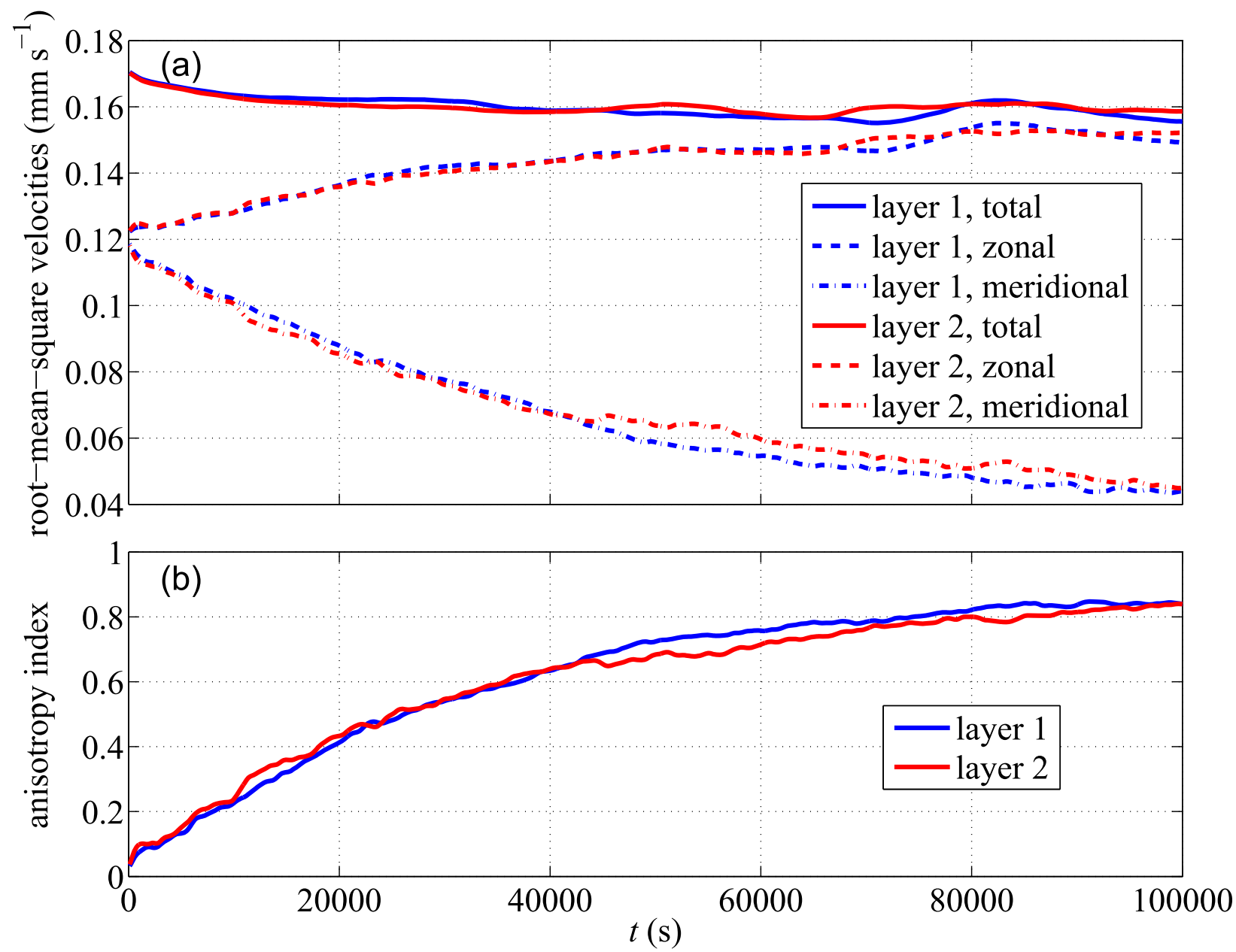

FIG. 6. Time series from simulation 11, showing the evolution of (a) the root-mean-square velocities in each layer, together with their zonal and meridional contributions, and (b) the anisotropy index. The spatial averaging is performed over the entire annular domain.

illustrative purposes. Figure 8a shows that the baroclinic streamfunction at a particular azimuthal angle oscillates quasi periodically with radius because of the baroclinic jets. Figure $8 \mathrm{~b}$ shows that the corresponding autocorrelation function also oscillates with radius, but with decreasing amplitude as the radial offset increases, which is the expected behavior for oscillations that are not perfectly periodic. We objectively diagnose the baroclinic jet width at this azimuthal angle by taking the first zero crossing of the autocorrelation function to be half of the jet width. This procedure diagnoses the jet width to be $0.73 \mathrm{~m}$. Figure $8 \mathrm{c}$ shows the azimuthal variation of the jet width, which is obtained by repeating the above procedure for each azimuthal angle. The jet width that is diagnosed in this manner exhibits substantial azimuthal variations. For example, the jet width is smaller at angles of around $135^{\circ}$ than $200^{\circ}$, which is consistent with visual inspection of Fig. 3f. Taking the mean and standard deviation over all azimuthal angles, the characteristic baroclinic jet width for this simulation at equilibrium is diagnosed to be $0.59 \pm 0.08 \mathrm{~m}$.

\section{b. Varying the parameters}

We will now analyze the final equilibrium states in all 15 simulations. The baroclinic streamfunction at the end of each simulation is shown in Fig. 9. Simulation 11, which is the case study that was analyzed in section 3a, appears in the top row of the right column. The values of $q_{0}, u_{\mathrm{rms}}$, and $L_{\mathrm{Rh}, \mathrm{bc}}$ each increase toward the bottom of the figure. Visual inspection indicates that the baroclinic jet width consequently increases, as expected. The values of $\Delta \rho$ and $L_{\text {Ro,bc }}$ each decrease toward the left of the figure. Visual inspection indicates that the baroclinic jets consequently become less zonally elongated and more undulatory, as expected. There is generally more evidence of robust anisotropy toward the top-right corner of the figure. It must be remembered when interpreting the figure that the structure of the jets has 


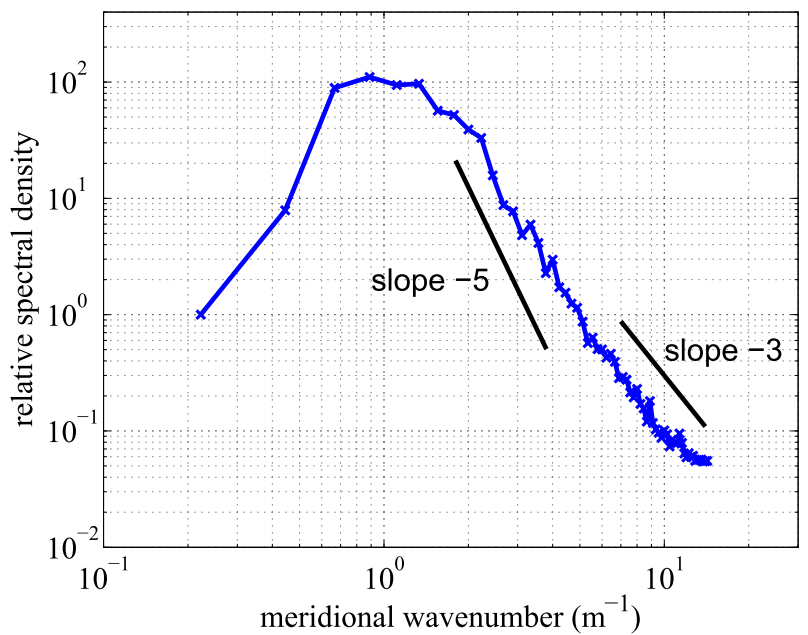

FIG. 7. Kinetic energy spectrum for the baroclinic zonal flow in simulation 11 . The quantity plotted is the kinetic energy of the baroclinic zonal flow, per unit mass and per unit meridional wavenumber. This quantity is a spectral density $\left(\mathrm{m}^{3} \mathrm{~s}^{-2}\right)$, but here it is normalized by the spectral density of the gravest meridional wave mode to yield a dimensionless relative spectral density. The spectrum is obtained by computing the squared absolute value of the radial Fourier transform of the baroclinic zonal velocity at $\theta=0^{\circ}$ between $r=a$ and $r=b$, and then averaging over time between 50000 and $100000 \mathrm{~s}$.

a temporal variability that is not captured in these snapshots and that coupling between the vertical modes may allow barotropic jets to leave a signature in the baroclinic flow, even if baroclinic jets are forbidden by the linear analysis in section $2 \mathrm{a}$.

The quantitative results from all 15 simulations are summarized in Table 3. In simulations $1-5$, the stratification is so weak that the baroclinic Rossby radius is only $12 \mathrm{~cm}$, implying that the phase speed limit of long baroclinic Rossby waves is only $\beta L_{\mathrm{Ro}, \mathrm{bc}}^{2}=0.62 \mathrm{~mm} \mathrm{~s}^{-1}$. This speed limit is comparable with the root-mean-square eddy velocities. Therefore, the equilibrated baroclinic flow is dominated by eddies and remains approximately isotropic. In simulations 6-10, the stratification is stronger and the baroclinic Rossby radius is increased to $37 \mathrm{~cm}$, implying that the phase speed limit of long baroclinic Rossby waves is increased to $6.2 \mathrm{~mm} \mathrm{~s}^{-1}$. This speed limit is at least a few times greater than the root-mean-square eddy velocities. Therefore, the equilibrated baroclinic flow displays undulatory zonal jets, with coexisting eddies disrupting the zonal coherence. The diagnosed jet widths display large azimuthal variations of $20 \%-50 \%$ about their azimuthal-mean values. In simulations $11-15$, the baroclinic Rossby radius is increased to $117 \mathrm{~cm}$, implying that the phase speed limit of long baroclinic Rossby waves is increased to $62 \mathrm{~mm} \mathrm{~s}^{-1}$. This speed limit is at least an order of magnitude greater than the root-mean-square eddy velocities. Therefore, the equilibrated baroclinic (a) baroclinic stream function $\left(10^{-4} \mathrm{~m}^{2} \mathrm{~s}^{-1}\right)$ at $\theta=200^{\circ}$

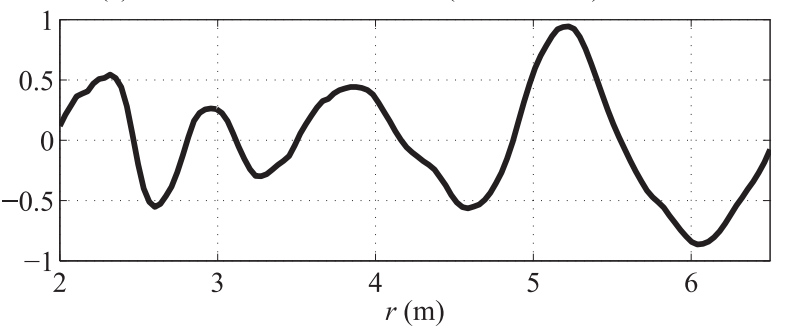

(b) autocorrelation function at $\theta=200^{\circ}$

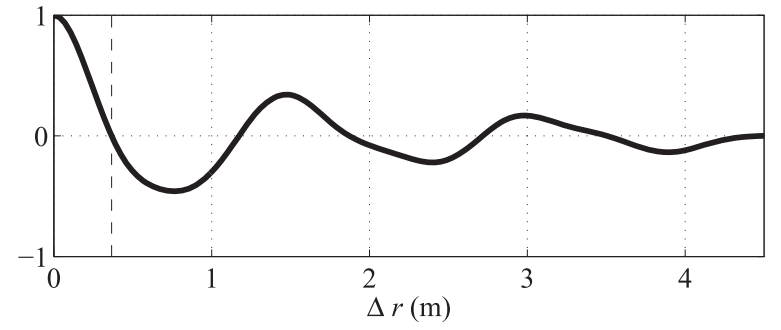

(c) baroclinic jet width $(\mathrm{m})$

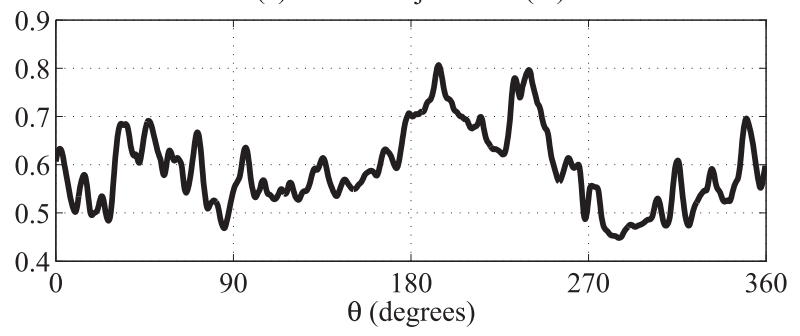

FIG. 8. Objective diagnosis of the baroclinic jet width after $100000 \mathrm{~s}$ in simulation 11, as derived from Fig. 3f. (a) The radial variation of the baroclinic streamfunction at $\theta=200^{\circ}$, which is approximately the " 8 o'clock" position. (b) The corresponding autocorrelation function, with its first zero crossing indicated by the dashed line and taken to be half of the jet width. (c) The azimuthal variation of the jet width, as diagnosed in this manner. The azimuthal angle $\theta=0^{\circ}$ refers to the " 3 o'clock" position, and $\theta$ increases in the anticlockwise direction.

flow is dominated by robust zonal jets, and the coexisting eddies are relatively weak. The diagnosed jet widths are now well defined, displaying small azimuthal variations of only $10 \%-30 \%$ about their azimuthal-mean values.

For simulations 11-15, a scatterplot of the diagnosed baroclinic jet width versus the theoretical baroclinic Rhines scale is shown in Fig. 10. If the theoretical scale were a perfect predictor of the jet width, then the points plotted would lie on a straight line of slope unity passing through the origin. Visual inspection indicates that the points are indeed consistent with linear behavior. The line of best fit has a slope of 2.5 instead of 1 , however, with a $95 \%$ confidence interval of $(2.2,2.8)$. The line of best fit has a $y$ intercept of -0.1 , with a $95 \%$ confidence interval of $(-0.3,0.1)$, but because this interval includes zero we conclude that the data are consistent with direct 

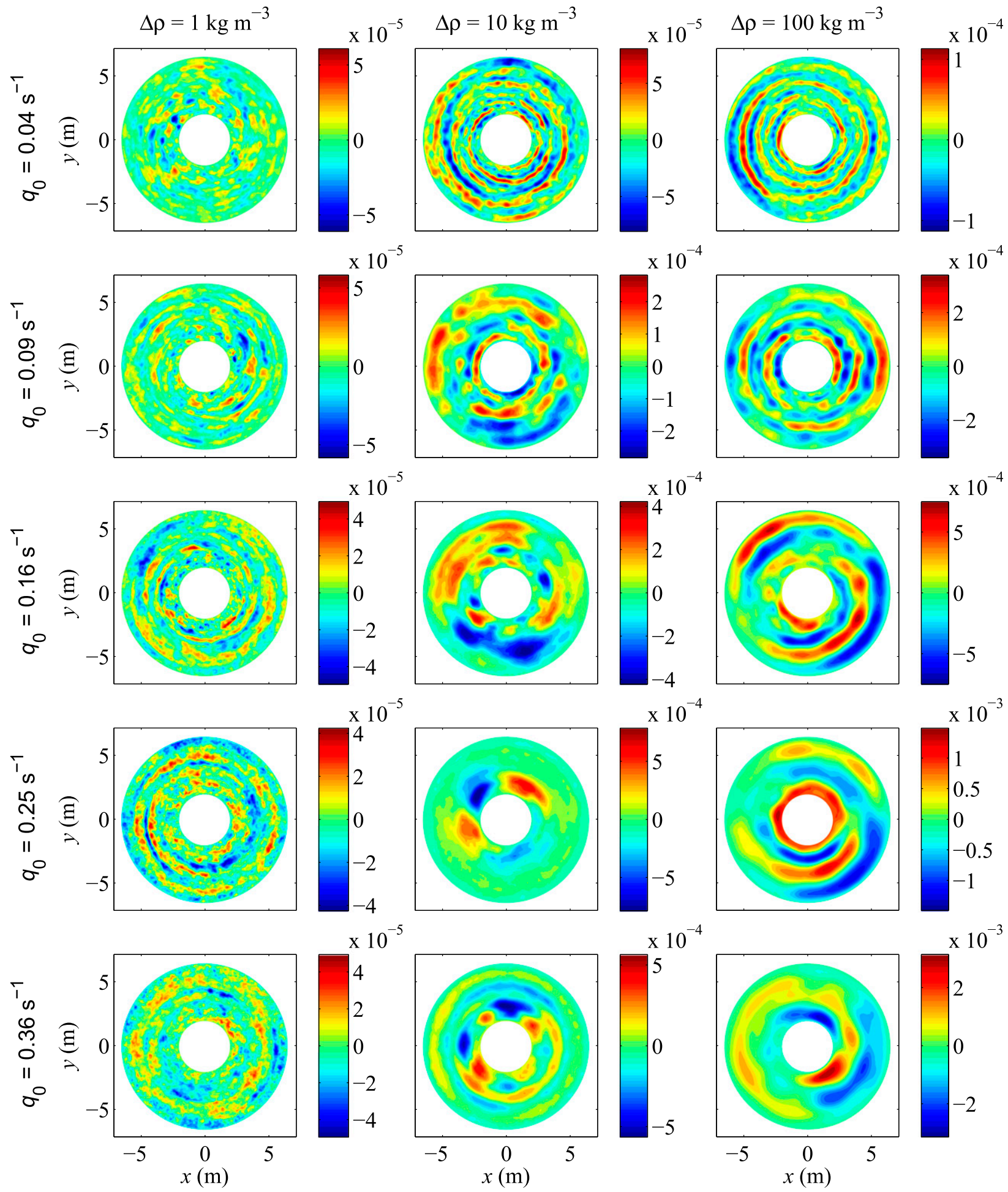

FIG. 9. Baroclinic streamfunction $\left(\mathrm{m}^{2} \mathrm{~s}^{-1}\right)$ after $100000 \mathrm{~s}$ for each combination of $\Delta \rho$ and $q_{0}$, that is, for simulations (left) $1-5$, (middle) 6-10, and (right) 11-15. 


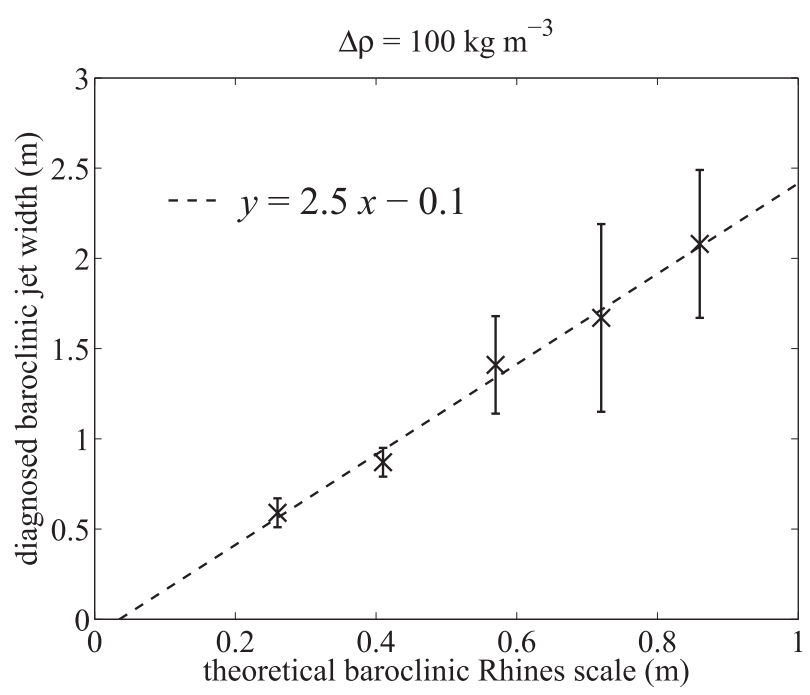

FIG. 10. Scatterplot from simulations 11-15, showing the relation between the diagnosed baroclinic jet width after $100000 \mathrm{~s}$ and the theoretical baroclinic Rhines scale given by (9). The error bars indicate the azimuthal variability of the diagnosed jet width about the azimuthal-mean value. The dashed line is the line of best fit, in the sense of minimizing the root-mean-square error.

proportionality. Therefore, the data suggest that the baroclinic jet width is given by a modified form of (9), namely:

$$
L_{\mathrm{Rh}, \mathrm{bc}}=2.5 \pi \sqrt{\frac{2 u_{\mathrm{rms}}}{\beta-u_{\mathrm{rms}} / L_{\mathrm{Ro}, \mathrm{bc}}^{2}}} .
$$

In summary, the width of the baroclinic zonal jets in the present simulations is $2.2-2.8$ times larger than would be expected from the usual definition of the Rhines scale, with a best estimate of 2.5 times larger. We note that Wordsworth et al. (2008) have also found that the Rhines scale significantly underestimates jet widths.

The Rhines scale calculated from (9) is not expected to be an exact match with the diagnosed jet width, for at least two reasons. First, the Rhines scale formula is obtained by equating the speeds of Rossby waves and turbulent eddies, which is not a rigorous derivation. Second, once the zonal jets have emerged from the turbulence, they create a background flow and thereby modify the Rossby wave dispersion relation. This feedback is neglected during the derivation of the Rhines scale formula. For these reasons, we do not expect exact quantitative agreement between the theoretical baroclinic Rhines scale and the diagnosed baroclinic jet width, and it is not even clear a priori which one should be larger. Nevertheless, the direct proportionality between these two quantities appears to be robust in Fig. 10.

\section{Summary and discussion}

This paper has investigated the dynamics of freely evolving baroclinic zonal jets in numerical simulations of a two-layer quasigeostrophic annulus with sloping topography. Small-scale eddies in the initial state were found to evolve through geostrophic turbulence and to accelerate zonally as they grew in horizontal scale, first isotropically and then anisotropically. If conditions allowed, this process led ultimately to the formation of zonal jets. The kinetic energy spectrum of the baroclinic zonal flow steepened from a -3 power law at small scales to a -5 power law near the jet scale. In a suite of $15 \mathrm{sim}$ ulations, the baroclinic Rossby radius and baroclinic Rhines scale were sampled by varying the stratification and root-mean-square eddy velocity, respectively. The conditions most favorable for producing multiple alternating baroclinic jets were found to be large baroclinic Rossby radius (i.e., strong stratification) and small baroclinic Rhines scale (i.e., weak root-mean-square eddy velocity).

The baroclinic jet width was diagnosed objectively and found to be 2.2-2.8 times larger than would be expected according to the usual definition of the Rhines scale, with a best estimate of 2.5 times larger. This result suggests a refinement to the derivation of the Rhines scales that was presented in section 2a. Specifically, the jet width in our study matches the length scale that would be obtained by equating the Rossby wave phase speed to $2.5^{2} \approx 6$ times the root-mean-square eddy velocity, instead of 1 times it. This empirical finding suggests that Rossby wave motions must be moving at speeds of approximately 6 times the turbulent eddy velocity in order to be capable of arresting the isotropic inverse energy cascade; moving at merely the same speed is not sufficient. A theoretical explanation for the factor of 2.5 is lacking at present, but could be a target for future work.

The present simulations may help with the interpretation of the laboratory experiments performed by Read et al. (2004, 2007). Numerical simulations have the potential to add value to the laboratory experiments in a number of different ways. First, the parameter space can be sampled relatively densely, whereas only three combinations of parameters could be explored in the laboratory. Second, simulations of very long duration can be run, whereas the duration of the laboratory experiments was limited to $6 \mathrm{~h}$. Third, the entire annular domain can be studied, whereas it was possible to capture laboratory images from a restricted azimuthal sector representing only $12 \%$ of the surface area of the annular channel. Finally, the baroclinic structure of the jets can be studied, whereas the jets in the laboratory were measured at only one vertical level. 
The Read et al. $(2004,2007)$ laboratory experiments had vertical density gradients of $1-2 \mathrm{~kg} \mathrm{~m}^{-4}$, giving a first baroclinic Rossby radius that was estimated to be approximately $10 \mathrm{~cm}$ (although the stratification varied in time under the influence of the saltwater forcing, starting from essentially neutral stratification). The laboratory experiments with the present rotation rate had root-mean-square eddy velocities of $2-3 \mathrm{~mm} \mathrm{~s}^{-1}$. Therefore, considering the suite of 15 simulations listed in Table 3, the closest match to the laboratory conditions is simulation 5. This simulation is not the most favorable for producing robust baroclinic jets, as shown in Fig. 9. This finding adds weight to the speculation by Read et al. (2004) that the meandering nature of their jets might have had something to do with subsidiary processes on the scales of the Rossby radii.

The Read et al. $(2004,2007)$ laboratory experiments had a maximum duration of $6 \mathrm{~h}$, which is $21600 \mathrm{~s}$ and corresponds to 540 rotation periods of the turntable. Figures 3-6 show that the simulated jets had not yet equilibrated after this time had elapsed. This finding adds weight to the speculation by Read et al. (2004) that their jets were still evolving toward the end of the laboratory experiments. The achievement of a dynamic equilibrium has been suggested to require at least 1000 rotation periods (Manfroi and Young 1999; Huang et al. 2001). Indeed, the present simulations extended to 2500 rotation periods, and the jets were still evolving noticeably until near the end.

A more detailed comparison with the Read et al. $(2004,2007)$ laboratory experiments would be of limited value, because there are several reasons why the present model is not directly comparable. First, the vertically continuous fluid in the laboratory experiments is replaced with two homogeneous layers in the model, capturing the first baroclinic mode but not the higher baroclinic modes. Second, the free upper surface in the laboratory experiments is replaced with a sloping rigid lid in the model. (However, the model's lid of slope $+s$ and base of slope $-s$ collectively produce the same potential vorticity gradient and topographic beta effect as the laboratory's base of slope -2s.) Finally, the turbulence in the laboratory experiments was continuously excited by small-scale forcing and dissipated by viscosity. This forced, dissipative regime is accessible to models (e.g., Huang and Robinson 1998; Scott and Dritschel 2012), but turbulence in the present model evolves freely, which is the approach that has been taken in several other studies of zonal jets (e.g., Yoden and Yamada 1993; Cho and Polvani 1996a).

We regard the analysis of the freely evolving turbulence simulations in this paper to be a prerequisite for studying the more complicated case of forced, dissipative turbulence. In the latter case, there is an additional natural length scale in the system. Defining $\epsilon$ to be the rate of energy input by the forcing, Maltrud and Vallis (1991) introduced $L_{\epsilon}=\left(\epsilon / \beta^{3}\right)^{1 / 5}$, which is the horizontal length scale at which the turbulence and Rossby wave frequencies are equal (see also Vallis and Maltrud 1993). The zonostrophy index, which is defined by $z=L_{\mathrm{Rh}} / L_{\epsilon}$, has been reported to be a key nondimensional parameter for discerning different flow regimes (Sukoriansky et al. 2007). For example, the strength of the jets has been reported to increase as $z$ increases (Scott and Dritschel 2012). It is possible to define a zonostrophic regime that occurs when $z>2.5$, whereas a friction-dominated regime occurs when $z<$ 1.5 and a transitional regime occurs when $1.5<z<2.5$ (Galperin et al. 2010). Di Nitto et al. (2013) have speculated that the weakness and meandering of the jets in the laboratory experiments of Read et al. (2004, $2007)$ is due to the relatively small value of the zonostrophy index $(z \sim 1.5)$. This speculation could be tested in future numerical simulations, by adding stochastic forcing and Ekman dissipation to the quasigeostrophic model studied here.

Acknowledgments. PDW is supported by a University Research Fellowship from the Royal Society under Grants UF080256 and UF130571. The research was supported in part by the National Science Foundation under Grant NSF PHY11-25915. The paper was written while PDW was a Visiting Fellow at the Isaac Newton Institute for Mathematical Sciences, Cambridge, United Kingdom. The paper was revised while PDW was visiting the Kavli Institute for Theoretical Physics, University of California, Santa Barbara, California. The hospitality of both institutes is gratefully acknowledged. The editor and two anonymous reviewers are thanked for their comments, which improved the paper.

\section{REFERENCES}

Afanasyev, Y. D., S. O'Leary, P. B. Rhines, and E. Lindahl, 2012: On the origin of jets in the ocean. Geophys. Astrophys. Fluid Dyn., 106, 113-137, doi:10.1080/03091929.2011.562896.

Asselin, R., 1972: Frequency filter for time integrations. Mon. Wea. Rev., 100, 487-490, doi:10.1175/1520-0493(1972)100<0487: FFFTI $>2.3 . \mathrm{CO} ; 2$.

Bakas, N. A., and P. I. Ioannou, 2013: On the mechanism underlying the spontaneous emergence of barotropic zonal jets. J. Atmos. Sci., 70, 2251-2271, doi:10.1175/JAS-D-12-0102.1.

Bastin, M. E., and P. L. Read, 1997: A laboratory study of baroclinic waves and turbulence in an internally heated rotating fluid annulus with sloping endwalls. J. Fluid Mech., 339, 173198, doi:10.1017/S0022112097005259.

, and - 1998: Experiments on the structure of baroclinic waves and zonal jets in an internally heated, rotating, cylinder of fluid. Phys. Fluids, 10, 374-389, doi:10.1063/1.869530. 
Bouchet, F., and J. Sommeria, 2002: Emergence of intense jets and Jupiter's Great Red Spot as maximum-entropy structures. J. Fluid Mech., 464, 165-207, doi:10.1017/S0022112002008789.

Cattaneo, F., and J. E. Hart, 1990: Multiple states for quasigeostrophic channel flows. Geophys. Astrophys. Fluid Dyn., 54, 1-33, doi:10.1080/03091929008208930.

Cho, J. Y.-K., and L. M. Polvani, 1996a: The emergence of jets and vortices in freely evolving, shallow-water turbulence on a sphere. Phys. Fluids, 8, 1531-1552, doi:10.1063/1.868929.

— winds in the atmospheres on the giant outer planets. Science, 273, 335-337, doi:10.1126/science.273.5273.335.

Di Nitto, G., S. Espa, and A. Cenedese, 2013: Simulating zonation in geophysical flows by laboratory experiments. Phys. Fluids, 25, 086602, doi:10.1063/1.4817540.

Dritschel, D. G., and R. K. Scott, 2011: Jet sharpening by turbulent mixing. Philos. Trans. Roy. Soc. London, A369, 754-770, doi:10.1098/rsta.2010.0306.

Dunkerton, T. J., and R. K. Scott, 2008: A barotropic model of the angular momentum-conserving potential vorticity staircase in spherical geometry. J. Atmos. Sci., 65, 1105-1136, doi:10.1175/ 2007JAS2223.1.

Galperin, B., S. Sukoriansky, and H.-P. Huang, 2001: Universal $n^{-5}$ spectrum of zonal flows on giant planets. Phys. Fluids, 13, 1545-1548, doi:10.1063/1.1373684.

- - H. Nakano, H.-P. Huang, and S. Sukoriansky, 2004: The ubiquitous zonal jets in the atmospheres of giant planets and Earth's oceans. Geophys. Res. Lett., 31, L13303, doi:10.1029/ 2004GL019691.

—_, S. Sukoriansky, and N. Dikovskaya, 2010: Geophysical flows with anisotropic turbulence and dispersive waves: Flows with a $\beta$-effect. Ocean Dyn., 60, 427-441, doi:10.1007/ s10236-010-0278-2.

Hovmöller, E., 1949: The trough-and-ridge diagram. Tellus, 1A, 62-66, doi:10.1111/j.2153-3490.1949.tb01260.x.

Huang, H.-P., and W. A. Robinson, 1998: Two-dimensional turbulence and persistent zonal jets in a global barotropic model. J. Atmos. Sci., 55, 611-632, doi:10.1175/1520-0469(1998)055<0611: TDTAPZ>2.0.CO;2.

- B. Galperin, and S. Sukoriansky, 2001: Anisotropic spectra in two-dimensional turbulence on the surface of a rotating sphere. Phys. Fluids, 13, 225-240, doi:10.1063/1.1327594.

Kwon, H. J., and M. Mak, 1988: On the equilibration in nonlinear barotropic instability. J. Atmos. Sci., 45, 294-308, doi:10.1175/ 1520-0469(1988)045<0294:OTEINB > 2.0.CO;2.

Lee, S., 2005: Baroclinic multiple zonal jets on the sphere. J. Atmos. Sci., 62, 2484-2498, doi:10.1175/JAS3481.1.

Limaye, S. S., 1986: Jupiter: New estimates of the mean zonal flow at the cloud level. Icarus, 65, 335-352, doi:10.1016/ 0019-1035(86)90142-9.

Maltrud, M. E., and G. K. Vallis, 1991: Energy spectra and coherent structures in forced two-dimensional and beta-plane turbulence. J. Fluid Mech., 228, 321-342, doi:10.1017/ S0022112091002720.

Manfroi, A. J., and W. R. Young, 1999: Slow evolution of zonal jets on the beta plane. J. Atmos. Sci., 56, 784-800, doi:10.1175/ 1520-0469(1999)056<0784:SEOZJO > 2.0.CO;2.

Marcus, P. S., and C. Lee, 1998: A model for eastward and westward jets in laboratory experiments and planetary atmospheres. Phys. Fluids, 10, 1474-1489, doi:10.1063/1.869668.

Maximenko, N. A., B. Bang, and H. Sasaki, 2005: Observational evidence of alternating zonal jets in the world ocean. Geophys. Res. Lett., 32, L12607, doi:10.1029/2005GL022728.
Panetta, R. L., 1993: Zonal jets in wide baroclinically unstable regions: Persistence and scale selection. J. Atmos. Sci., 50, 2073-2106, doi:10.1175/1520-0469(1993)050<2073: ZJIWBU $>2.0 . \mathrm{CO} ; 2$.

Read, P. L., Y. H. Yamazaki, S. R. Lewis, P. D. Williams, K. MikiYamazaki, J. Sommeria, H. Didelle, and A. Fincham, 2004: Jupiter's and Saturn's convectively driven banded jets in the laboratory. Geophys. Res. Lett., 31, doi:10.1029/ 2004GL020106.

J. Sommeria, and H. Didelle, 2007: Dynamics of convectively driven banded jets in the laboratory. J. Atmos. Sci., 64, 40314052, doi:10.1175/2007JAS2219.1.

Rhines, P. B., 1975: Waves and turbulence on a beta-plane. J. Fluid Mech., 69, 417-443, doi:10.1017/S0022112075001504.

Saito, I., and K. Ishioka, 2013: Angular distribution of energy spectrum in two-dimensional $\beta$-plane turbulence in the long-wave limit. Phys. Fluids, 25, 076602, doi:10.1063/ 1.4813808 .

Sanchez-Lavega, A., J. F. Rojas, and P. V. Sada, 2000: Saturn's zonal winds at cloud level. Icarus, 147, 405-420, doi:10.1006/ icar.2000.6449.

Scott, R. K., 2010: The structure of zonal jets in shallow water turbulence on the sphere. IUTAM Symposium on Turbulence in the Atmosphere and Oceans, D. Dritschel, Ed., IUTAM Bookseries, Vol. 28, Springer Netherlands, 243-252.

— , and D. G. Dritschel, 2012: The structure of zonal jets in geostrophic turbulence. J. Fluid Mech., 711, 576-598, doi:10.1017/ jfm.2012.410.

Slavin, A. G., and Y. D. Afanasyev, 2012: Multiple zonal jets on the polar beta plane. Phys. Fluids, 24, 016603, doi:10.1063/ 1.3678017.

Smith, C. A., K. G. Speer, and R. W. Griffiths, 2014: Multiple zonal jets in a differentially heated rotating annulus. J. Phys. Oceanogr., 44, 2273-2291, doi:10.1175/JPO-D-13-0255.1.

Sommeria, J., S. D. Meyers, and H. L. Swinney, 1989: Laboratory model of a planetary eastward jet. Nature, 337, 58-61, doi:10.1038/337058a0.

Sukoriansky, S., B. Galperin, and N. Dikovskaya, 2002: Universal spectrum of two-dimensional turbulence on a rotating sphere and some basic features of atmospheric circulation on giant planets. Phys. Rev. Lett., 89, 124501, doi:10.1103/PhysRevLett.89.124501.

_ - N. Dikovskaya, and B. Galperin, 2007: On the arrest of inverse energy cascade and the Rhines scale. J. Atmos. Sci., 64, 3312-3327, doi:10.1175/JAS4013.1.

Thompson, A. F., and W. R. Young, 2007: Two-layer baroclinic eddy heat fluxes: Zonal flows and energy balance. J. Atmos. Sci., 64, 3214-3231, doi:10.1175/JAS4000.1.

Vallis, G. K., and M. E. Maltrud, 1993: Generation of mean flows and jets on a beta plane and over topography. J. Phys. Oceanogr., 23, 1346-1362, doi:10.1175/1520-0485(1993)023<1346: GOMFAJ $>2.0 . \mathrm{CO} ; 2$.

Williams, P. D., 2009: A proposed modification to the RobertAsselin time filter. Mon. Wea. Rev., 137, 2538-2546, doi:10.1175/2009MWR2724.1.

—_ 2011: The RAW filter: An improvement to the RobertAsselin filter in semi-implicit integrations. Mon. Wea. Rev., 139, 1996-2007, doi:10.1175/2010MWR3601.1.

— 2013: Achieving seventh-order amplitude accuracy in leapfrog integrations. Mon. Wea. Rev., 141, 3037-3051, doi:10.1175/MWR-D-12-00303.1.

- , P. L. Read, and T. W. N. Haine, 2003: Spontaneous generation and impact of inertia-gravity waves in a stratified, 
two-layer shear flow. Geophys. Res. Lett., 30, 2255, doi:10.1029/ 2003 GL018498.

— T. W. N. Haine, and P. L. Read, 2004: Stochastic resonance in a nonlinear model of a rotating, stratified shear flow, with a simple stochastic inertia-gravity wave parameterization. Nonlinear Processes Geophys., 11, 127-135, doi:10.5194/ npg-11-127-2004.

,-- , and -2005 : On the generation mechanisms of shortscale unbalanced modes in rotating two-layer flows with vertical shear. J. Fluid Mech., 528, 1-22, doi:10.1017/S0022112004002873. , - - - S. R. Lewis, and Y. H. Yamazaki, 2009: QUAGMIRE v1.3: A quasi-geostrophic model for investigating rotating fluids experiments. Geosci. Model Dev., 2, 13-32, doi:10.5194/gmd-2-13-2009.

—, P. L. Read, and T. W. N. Haine, 2010: Testing the limits of quasi-geostrophic theory: Application to observed laboratory flows outside the quasi-geostrophic regime. J. Fluid Mech., 649, 187-203, doi:10.1017/S0022112009993405.

Wordsworth, R. D., P. L. Read, and Y. H. Yamazaki, 2008: Turbulence, waves, and jets in a differentially heated rotating annulus experiment. Phys. Fluids, 20, 126602, doi:10.1063/1.2990042.

Yoden, S., and M. Yamada, 1993: A numerical experiment on twodimensional decaying turbulence on a rotating sphere. $J$. Atmos. Sci., 50, 631-644, doi:10.1017/S0022112091002720. 